\title{
A Pain Reporting Platform for Adolescents with Sickle-Cell Disease
}

\author{
Kevin A. Gary \\ Arizona State University \\ Mesa, AZ USA \\ kgary@asu.edu \\ Zenaide Quezado \\ National Institutes of Health \\ Bethesda, MD USA \\ zquezado@cc.nih.gov
}

\author{
Pooja Rallabhandi \\ Arizona State University \\ Mesa, AZ USA \\ Pooja.Rallabhandi@asu.edu \\ Kevin Cleary \\ Childrens National Health System \\ Washington, D.C. USA \\ KCleary@childrensnational.org
}

\begin{abstract}
This paper presents the latest version of the Pain Reporting and Management mHealth Platform for adolescent Sickle Cell Disease, developed in collaboration by Arizona State University and the Children's National Health System. This platform supports a cross-platform mHealth app, reporting and task management API, and portal dashboard for care provider monitoring. Extending our prior work, the latest version adds enhanced app features (games, power-ups, badges, notifications) to promote sustained adherence to the reporting protocol, and enhanced reporting features for providers that track high fidelity compliance measures and aggregate outcome scores. This paper summarizes the architecture and principle features of the platform, and presents data supporting improved compliance.
\end{abstract}

\section{Introduction}

According to the World Health Organization (WHO) Global Observatory for eHealth [1], mhealth is a "medical and public health practice supported by mobile devices, such as mobile phones, patient monitoring devices, personal digital assistants (PDAs), and other wire-less devices." Mobile devices have the advantage of being portable, constantly connected via the internet, giving an individual a sense of privacy (in the sense of avoiding the stigma of being seen entering a clinic, not in the data privacy sense), being able to act like sensors for monitoring and collecting an individual's daily activity data. These factors have made mHealth popular over the period [2-7].

Ashburn and Staats [8] define chronic pain as "pain that persists for longer than expected time frame for healing or pain associated with progressive, nonmalignant disease". Patients in this condition commonly experience depression, sleep disturbance, fatigue and decreased overall physical and mental functioning. These patients frequently require appropriate medical care to address their pain experience. Dansie and Turk [9] performed an assessment on patients with chronic pain. They suggest clinicians require information about a patient's medical history, symptoms causing chronic pain and any patterns in the patient behavior over the time for making decisions regarding chronic pain treatment. The authors mention that as there is no specific instrument that can provide an objective quantification of the extent or severity of pain experienced by the patient, it can only be assessed based on verbal and non-verbal communication with the patient. This form of data collection is termed as self-reported or patientreported data. There are benefits to using this method for data collection. Information collected in this manner can be considered more accurate as it is directly being reported by the person with pain, avoiding human error in the data collection and transcription process, thus making it reliable for the clinicians to use for suggesting various treatments.

To validate self-reported data collected from patients, the National Institutes of Health (NIH) created the Patient-Reported Outcomes Measurement Information System (PROMIS) in late 2004. PROMIS consists of valid, generalizable item banks used to measure key symptoms and health concepts applicable 
to a range of chronic conditions, enabling efficient and interpretable clinical trial and clinical practice applications of patient-reported outcomes (PROs). Several PROMIS instruments deal with pain reporting and comorbidities that are associated with pain.

Arizona State University (ASU), in collaboration with the Pain Management Care Complex, a part of the Sheikh Zayed Institute's Pain Medicine Initiative at Children's National Health System (CNHS) in Washington, D.C., developed an mHealth platform for monitoring pain-related clinical outcomes. Prior to this platform, the clinic relied upon paper-based survey instruments to collect data regarding patient pain intensity and burden. The main observed problem was a lack of patient compliance, as not many patients would visit the clinic as per the schedule to provide this data in paper form. Additionally, care providers lacked an efficient reporting mechanism to track patient compliance and PROMIS aggregate scores. To address these issues, the Sickle Cell Disease (SCD) Pain Reporting \& Management Platform was developed. The platform has gone through multiple development iterations, with the latest version used in a 76-patient clinical trial. This paper presents the platform, and discusses key features enabling sustained patient engagement and care provider efficiency. Patient engagement results are also discussed.

\section{Background}

Our focus is on pain treatment and management for children and adolescents with SCD, consistent with the mission of CNHS. Our technical approach for mHealth has been to create a platform leveraging APIdriven mHealth applications and tools to increase patient adherence to self-reporting tasks. Without patient adherence to self-reporting tasks, the data collected is insufficient for study in the clinical context. This section provides a brief overview of relevant work and motivating context on SCD and engagement mechanisms in mHealth.

\subsection{Sickle Cell Disease}

Sickle cell disease (SCD) is a condition which causes multiple disorders, most prevalently chronic hemolytic anemia (anemia caused by breakdown of Red Blood Cells), dactylitis (inflammation of bones in hands and toes) and vaso-occlusive crises. SCD is characterized by an abnormal shape of the red blood cells, which appear like bent sickles. SCD occurs in people who inherit (recessively) genes that produce abnormal hemoglobin molecules, which in fact gives rise to abnormal "sickle" red blood cells and various other disorders associated with SCD [10].

Pain in SCD can be broadly categorized into two types, acute pain crises and chronic pain. Acute pain crises can be mostly attributed vaso-occlusive crises (VCO), which refers to obstruction of blood flow due to abnormal "sickle" cells. VCO leads to ischemic injuries, dactylitis, priapism, abdominal pain, acute chest syndrome. Chronic pain refers to prolonged periods of pain and can be attributed to the disrupted or disordered physiological process (pathophysiology) inherent to SCD or patient reported pain with no obvious causes. According to Ballas [11], untreated acute pain crises can lead to chronic pain in patients suffering from SCD.

The CDC estimates there are 90,000 to 100,000 Americans suffering from SCD and African Americans and Hispanics are especially a risk. It is estimated that 1 in every 500 African Americans and 1 in every 36,000 Hispanic Americans are born with SCD.

Pain is often considered the most prevalent cause of re-hospitalizations in patients suffering from Sickle Cell Disease (SCD). In one study it was estimated that $78 \%$ people reported admission and $70 \%$ people reported re-admission to a hospital in patients suffering from SCD [12]. In many cases untreated acute pain crises can lead to chronic pain conditions [11].

One study suggested that hospital readmission rates within 30-days was as high as 30 patients in 100 cases. Among the patients the most prevalent cause for admission (78\%) and re-admission (70\%) was found to be pain. The same study also found that the greatest risk factor for re-admission was lack of follow up of outpatients with the hematologists [12]. A different study shows that almost $10.2 \%$ of the cases they studied were re-hospitalized within 14 days and almost $17 \%$ were re-hospitalized within 30 days. It was concluded that follow up with outpatients has a reduced number of re-hospitalizations in patients with SCD [13]. Outpatient management is regarded as one of the key aspects in healthcare for SCD, as this will help the healthcare providers to decide on a course of treatment or intervention [14].

It is important to note that the Health-Related Quality of Life (HRQL) is significantly lower in SCD patients. SCD patients suffer significantly in the HRQL physical functioning domain, and pain is attributed as the dominant cause. Thus, HRQL measurement must be included in the daily care of patients suffering from SCD [15]. It was also found that there are several issues that plague the patients suffering from SCD, including fear of an early death, uselessness, helplessness and inability to interact with others [16].

Pain management in outpatients can be challenging, in fact it was found that adolescents have 
trouble keeping up with clinical appointments due to conflicting schedules, bad health or forgetting the appointment [17]. Our goal is to make the process of pain management a very easy and efficient through the use of mobile and web technologies. The PROMIS $₫$ questionnaires have been used to measure the patient reported outcomes (PRO) on patient's health status and general quality of life.

Mobile systems give unprecedented accessibility to patients because most adolescents have access to a cell phone or tablet these days. It is estimated that almost $73 \%$ of American teenagers have access to a smart phone, with $92 \%$ of teenagers reporting accessing the Internet daily out of which $24 \%$ "go online almost constantly" [18]. This makes mHealth a viable solution for self-reporting comorbidities of pain instead of making patients frequently travel to a clinic.

\section{2 mHealth Engagement}

The theoretical framework for our work in patient engagement impacting adherence to the target clinical protocol is based on the work of Ryan and Deci [19] in defining intrinsic and extrinsic motivation. Intrinsic motivation is doing an activity for its inherent satisfactions than for some external consequences. When a person is intrinsically motivated, s/he is moved to act for the perceived benefits to oneself rather than because of external prods, pressures or rewards. Extrinsic motivation pertains to an activity done to attain some external separable outcome. When a person is extrinsically motivated, s/he performs a specific task to receive either recognition or a reward or price for successful completion of a task.

Researchers have carried out studies to find out factors facilitating or undermining intrinsic and extrinsic motivations for improving user engagement (cf. [20,21,22]). In the SCD app, intrinsic motivational features are few; the principal mechanism is a "Did You Know" style tip that a user is shown each time $\mathrm{s} /$ he arrives at the landing page of the app. There are several extrinsic motivational features based on gamification strategies - games, powerups and badges.

Gamification is the embedding of gameplay techniques into non-game activities [2]. This is slightly more encompassing than games themselves, as it includes reward mechanisms like badges. Gamification's goal is to increase user engagement by using game-like techniques to make people feel more ownership and purpose towards the tasks engaged [3]. Games themselves may be embedded, meaning the act of playing the game is the protocol activity [23] making them part of intrinsic motivation, or they may be external to the protocol, in which gameplay is held as a reward for adhering to the task schedule of the protocol. In the case of the SCD app, two open source games were taken and integrated into the application and gameplay was allowed as an extrinsic reward for completing PROMIS surveys. Further, badges were awarded for consecutive completions of daily surveys and weekly PROMIS surveys. Badges motivate continued user engagement in a task, which increases time spent on the task and supports skill acquisition through performance [24]. Typically they are used as a visible external symbol of task completion or skill acquisition [2] shared with a social circle of some form. For example, in learning platforms badges may represent a competency gained through training, which in turn are shared on an external website (e.g. a gradebook or a portfolio page such as on LinkedIn). However in the SCD platform in this study we did not want to externally share such symbols as it could lead to personally identifying information (PII), and our protocol was to use de-identified users. Instead, earned badges provided a form of positive feedback, and additionally could be cashed in as "power-ups" in the games (e.g. extra lives for the user agent in the game, or easier starting points in the games). Screenshots of integrated games and badge awards are in Figure 3. In our experience in multiple gamified mHealth apps, we have found evidence that they may actually serve as a distraction instead of proper motivator [25].

\section{Platform Implementation}

Our solution for an effective pain management system was to develop a web/mobile application and portal based on NIH's PROMIS $®$ questionnaires. The SCD Pain Reporting and Management Platform offers the following key features:

1. Accessibility through the use of mobile systems.

2. Reliability in Patient Reported Outcomes through the use of PROMIS ${ }^{\circledR}$.

3. Multiplatform approach (Android, iOS, Web) to target as many users as possible.

Enhanced engagement features (games, powerups, badges, notifications) to encourage sustained compliance to the reporting protocol.

The SCD Pain Reporting and Management platform is comprised of an mHealth app available on Android, iOS, or a standard Web browser, an API (Application Program Interface), a care provider portal, and a MySQL database. Patients recruited to use the app are provisioned by a care provider via the portal, given a de-identified personal code, and shown how to install and use the app. Settings for the app include the location of the API, which controls the scheduling of activities that appear in the app according to a protocol's schedule. Figure 1 shows the key components and interactions in the platform. 


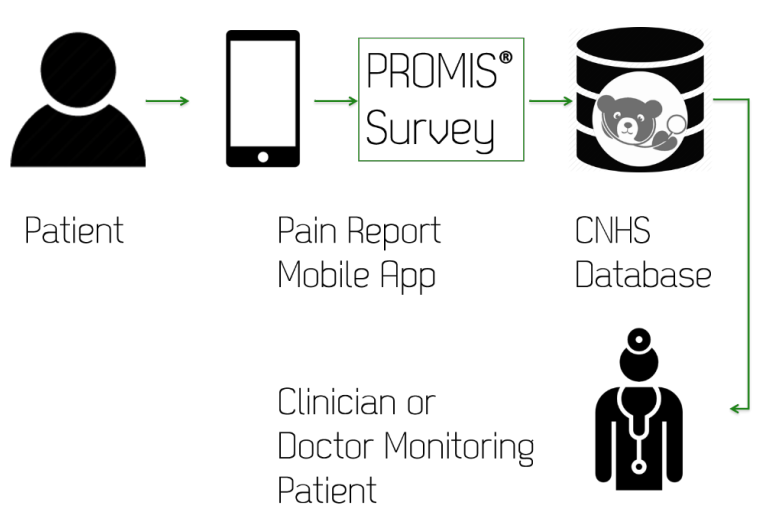

Figure 1. Platform Workflow Overview

The mHealth app at its core is a web application with native wrappers that enable it to run on smart phones as a native application. The web application, running in a webview on the mobile device, uses the AngularJS and Bootstrap frameworks. The native wrappers are developed for both Android and iOS, in which a system of local notifications was also implemented. The API was developed in Java using the open source Jersey framework and is based on REST principles. The care provider portal is written in NodeJS. The backend database is a MySQL relation database using embedded JSON to store metadata to control activity delivery and rendering. The architecture view of the system is shown in Figure 2.

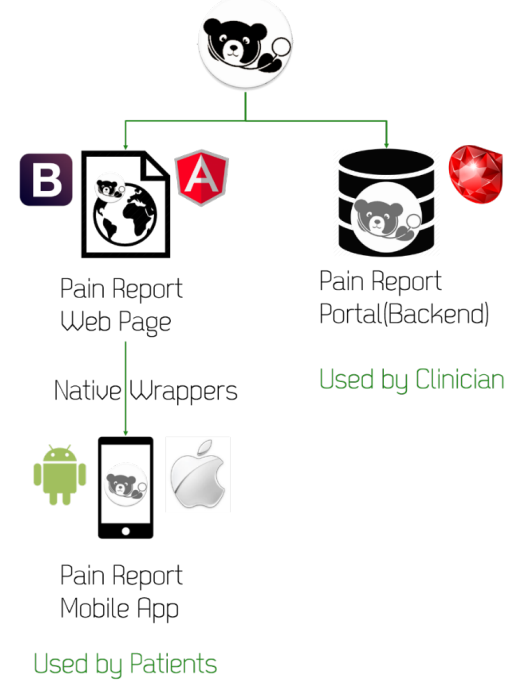

Figure 2. Platform Architecture View

The API-driven nature of the platform is innovative. It provides for a form of "remote control" of the app. Clinicians are able to manipulate the sets of activities delivered to specific individual or groups of end users, and new composite activities may be created by aggregating existing activities already defined for a particular step in the protocol. For example, the app delivers 4 PROMIS short forms in a weekly activity; these 4 short forms are aggregated into one activity delivery through the composite activity mechanism, which enables randomizing delivery of short form questions to try and eliminate bias, while keeping the question identified to their respective groups for later data collection and analysis.

\section{1 mHealth App}

Patients interact with the mHealth app by responding to a notification issued on the smartphone that a protocol task is due. The app determines a task is due by being informed by the API that the patient should complete a task. When the app opens it brings the user to a landing page that indicates the set of tasks the patient should perform (Figure 3a).

Two versions of the app were distributed for evaluation; an "ordinary" version that simply allowed the patient to complete the protocol tasks - complete PROMIS surveys (Figure 3c) and some additional questionnaires (Figure 3d), and an "enhanced" version that included two open source games (Figure 3e), a badging system (Figure 3f), and "tips" informing the patient why it was important to continue engaging in this study (Figure 3b) shown when the app starts up.

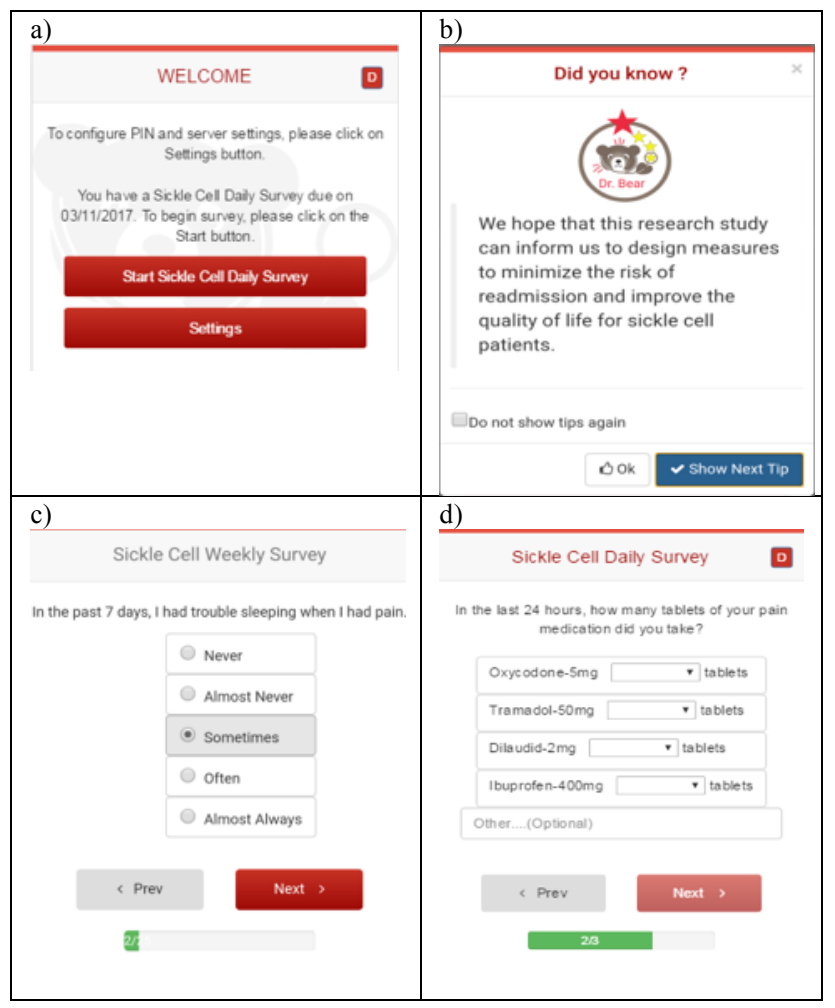




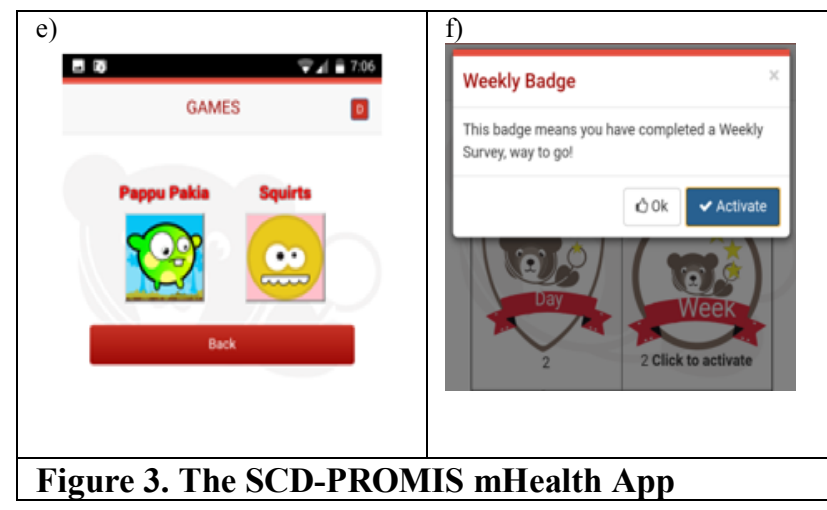

Section 4 discusses patient recruitment and enhanced versus non-enhanced distribution strategies, while section 5 presents results from introducing the enhanced features.

\subsection{REST API}

The REST API provide endpoints for accessing resource definitions in the domain. These include resources such as Patients, Trials, Surveys, and Games. The key workflow abstraction is an Activity, which may be a Composite Activity or a Base Activity. A Base Activity corresponds to a task a patient actually performs, like completing a PROMIS short form or playing a game. A Composite Activity combines Base Activities and other Composite Activity definitions to form a tree; the Composite Activity then defines the rules under which to deliver its subtrees.

For example, one business rule in the system is to deliver a PROMIS short form in its entirety. A short form is typically 6-8 Likert-scale questions. But typically, a patient is asked to complete 4 short forms each week in a single sitting (interaction with the app). To combine the 4 short forms randomly, a Base Activity is created for each short form task, and a Composite Activity is created to combine the 4 into one deliverable serialized activity instance of the combined surveys.

\subsection{Care Provider Portal}

The care provider portal provides basic study management features, which an ability to drill-down to individual patients. Data export features allow for offline exploration of detailed compliance and PROMIS survey result data. The top-level view of a study or clinical trial provides a summary view of compliance (Figure 4). A patient drill-down view shows compliance data (Figure 5) and a PROMIS scores chart (Figure 6) showing aggregate score for each short form based on the PROMIS scoring algorithm.
The Compliance Report (Figure 5) demonstrates one small way mHealth can change a traditional concept. Task adherence is usually a binary decision the patient either completes the task or does not; but a self-reporting mHealth app such as ours allows for software measures of adherence, where we can track if the patient is completing the task when initially asked or at the last minute, which may speak to the level of intrinsic motivation. Likewise, the Scores chart provides a visual way to correlate short form scores with additional measures collected by the app, such as medication adherence and pain intensity levels.

At present only clinicians and their staffs are given access to the portal. They use it to enroll new patients, export data, deactivate patients who have dropped out of the study, and track patient adherence and scores during the period of the user's enrollment in the clinical trial. Future plans call for parent and perhaps patient views of the reports available in the portal as a means to enhance intrinsic motivation.

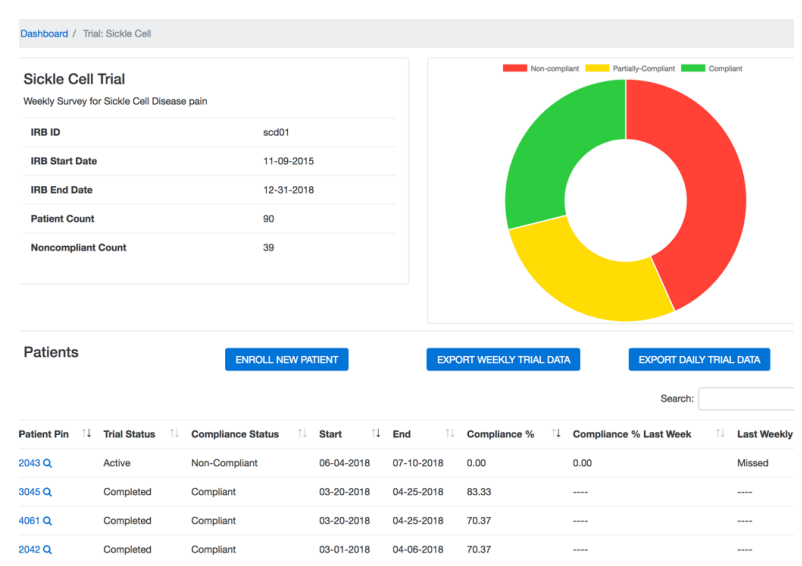

Figure 4. Portal trial-level overview screen

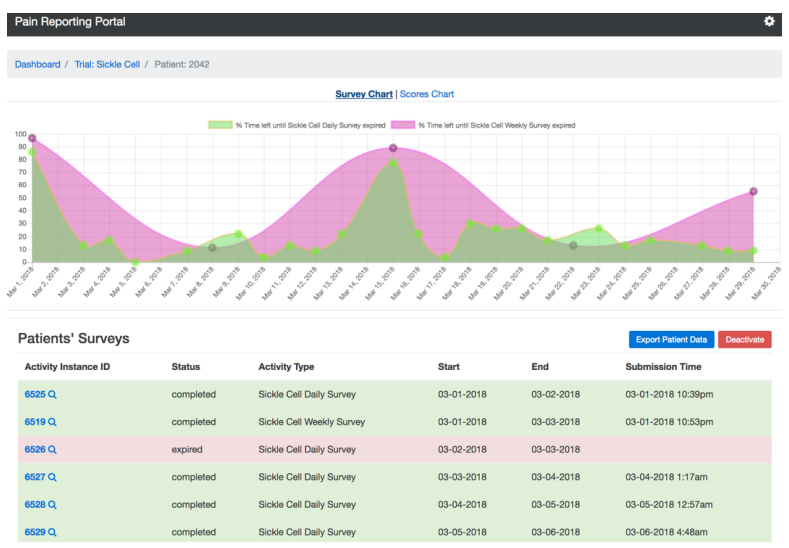

Figure 5. Compliance Report for Individual Patient 


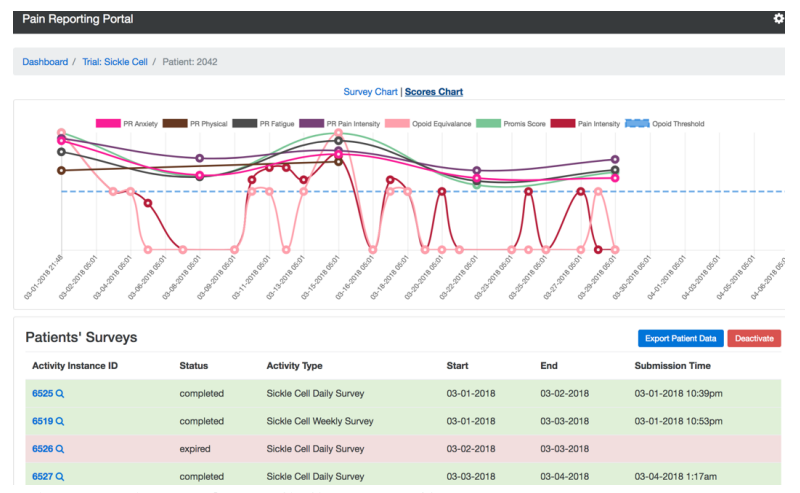

Figure 6. PROMIS Scores Chart

\section{Methodology}

Patients were recruited for the clinical trial under IRB approval at the Children's National Health System (CNHS) in Washington D.C. Patients who visited the pain clinic at CNHS were recruited if they were between 8 and 21 years old and were diagnosed with SCD. Recruited patients in the 8-17 year-old range must also have had a parent indicating consent, and the parent was given a variation of the SCD app to observe and report on her/his child. Upon obtaining informed consent, the patient (and parent if $<18$ year old) were given 4-digit secret access codes used to identify their response and activity with the app through the API. The access codes could only be re-unified with the patient identity outside the scope of the SCD platform and only by the clinical PI and her assigned staff. Patients (and parents), with the assistance of clinical staff, loaded the application via the respective app store (Google Play or iTunes) based on the operating system of their personal device (Android or iOS). Clinical staff led the user(s) through a first use of the app, demonstrating features and having the patient (and parent if applicable) take a first daily and weekly survey. Questions were resolved before the users left the clinic. No users came back and indicated at a later time that the app was unstable or too confusing to use effectively.

Patients were enrolled for a period of 36 days and expected to complete a daily survey each day, and a weekly survey each week (a total of six weekly surveys). Clinicians used the portal to track patient activity and survey responses. User interactions were logged throughout the application and sent to through the API to the server, except for events within the integrated games. If a patient was readmitted to the hospital during the 36-day period, then the affected users were deactivated from the study, though their recorded data remained.

Initially all users were given a non-enhanced version of the application, and later the assignment of the enhanced versus non-enhanced version of the app alternated for each new recruit. Parents did not receive an enhanced version of the app.

As the portal was only used by a clinician, her staff assistant, and supporting technologists, it did not impact patient engagement and adherence to the protocol and hence is not included in the results discussed in the next section.

\section{Results}

The SCD Pain Reporting and Management Platform has been used for over a year to support a clinical trial on subjects aged 8-21 with SCD. The goal of the study is to try and determine self-reporting factors that may predict hospital readmission in a 30day time window. In order to construct such a model the platform must be able to support data collection, and motivate patients to complete their tasks. This is an identified problem for mHealth apps - how to prevent notification or alarm fatigue, as users become bored with apps in a relatively short timespan. Our results with prior versions of this app and other apps we have developed show patients may have a significant drop in engagement after 2 weeks of using the technology. Our goal was to show that an API-driven on-demand app, plus an enhanced version utilizing games, powerups, and badges, could show sustained engagement.

We compiled the compliance data for daily and weekly PROMIS survey activities in the app for patients that were active in the trial for at least $50 \%$ of the time period (18 days of a 36-day protocol). This resulted in 76 patients, 23 of whom received the enhanced version of the app. Further, the 76 patients were split into 3 categories: children (ages 8-17, 27 patients), parents of children 8-17 (26 parents), and adults (ages 18-21, 23 patients). We had one more child than parent as we discarded one parent user who never used the platform at all. Children and adults (the actual patients) could receive the enhanced version of the app (randomly assigned) but parents could not. Parents received the parent proxy versions of the corresponding short forms given to children (pain, anxiety, fatigue, and physical functioning).

We were interested in two main questions:

1) Does compliance significantly drop off after 2 weeks of study participation?

2) Did the enhanced version encourage better compliance through sustained app engagement? 


\section{Daily Compliance}

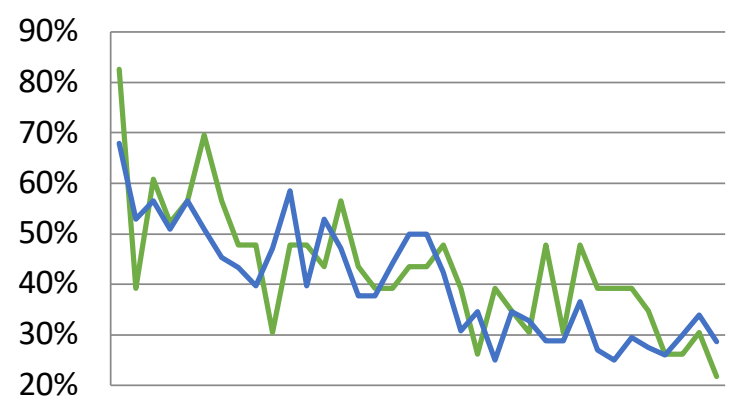

$\begin{array}{llllllllllll}1 & 4 & 7 & 10 & 13 & 16 & 19 & 22 & 25 & 28 & 31 & 34\end{array}$

Figure 7. Daily Survey Compliance

Figure 7 shows the rate of survey compliance over the course of the study for survey activities given on a daily basis. Green is the enhanced version of the app, and blue the non-enhanced version. The chart shows that the enhanced version generally had higher compliance rates, though not always; and that compliance did show a downward trend the further into the trial, though did not "fall off a cliff" after week 2.

The raw data for daily survey activities is given in Table 1 . The overall compliance rate for the app was $41 \%$, and both adults and children showed higher compliance using the enhanced version of the app.

Table 1. Raw compliance data for daily surveys

\begin{tabular}{|c|c|c|c|c|c|c|c|c|c|}
\hline & \multicolumn{3}{|c|}{ Overall } & \multicolumn{3}{|c|}{ Enhanced } & \multicolumn{3}{|c|}{ Non-enhanced } \\
\hline adult & 270 & 527 & $34 \%$ & 119 & 185 & $39 \%$ & 151 & 342 & $31^{\circ}$ \\
\hline child & 435 & 537 & $45 \%$ & 237 & 267 & $47 \%$ & 198 & 270 & $42^{\circ}$ \\
\hline parent & 410 & 526 & $44 \%$ & & & erall: & 1115 & 1590 & $41^{\circ}$ \\
\hline
\end{tabular}

Weekly survey results (Figure 8) were a bit more consistent than daily survey results.

\section{Weekly Compliance}

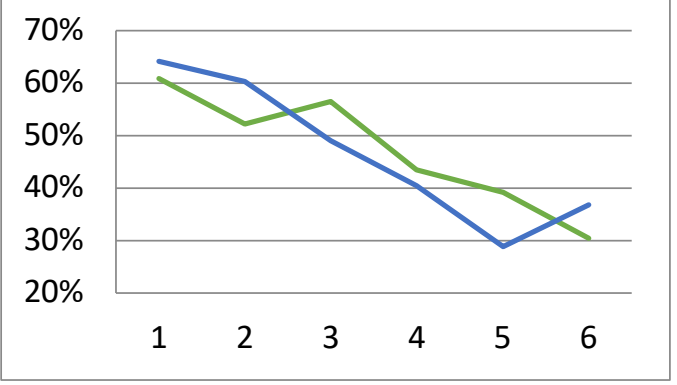

Figure 8. Weekly Survey Compliance
The downward trend for weekly surveys is still apparent, and consistent with the slope of the daily surveys (Figure 7). Strangely there is an upward tick for non-enhanced app users in the final week of the study that does not appear for enhanced users. Again, compliance degrades as is consistent with the literature, though there is no sharp dropoff. This provides some evidence that the fatigue effect of mHealth app usage is moderated by the set of enhanced engagement features embedded in the app.

Table 2 gives the raw weekly survey compliance data. It is important to understand that weekly activities are longer and (obviously) more infrequent than daily activities. One would expect weekly compliance rates to be lower because of this but we did not find this to be the case. We are not certain yet whether this is due to some of the enhanced features being weighted more heavily toward weekly activities, or if perhaps daily survey activities incurred some task fatigue.

It is interesting to note compliance actually went up slightly for non-enhanced adult patients. This may indicate games were not age-appropriate for older patients. Adult patients showed much higher compliance rates in weekly activities than daily activities yet children and parents were consistent.

Table 2. Raw compliance data for weekly surveys

\begin{tabular}{|l|r|r|r|r|r|r|r|r|r|}
\hline & \multicolumn{3}{|c|}{ Overall } & \multicolumn{3}{c|}{ Enhanced } & \multicolumn{3}{c|}{ Non-enhanced } \\
\hline adult & 62 & 70 & $47 \%$ & 22 & 29 & $43 \%$ & 40 & 41 & $49 \%$ \\
\hline child & 79 & 83 & $49 \%$ & 43 & 41 & $51 \%$ & 36 & 42 & $46 \%$ \\
\hline parent & 70 & 86 & $45 \%$ & & \multicolumn{2}{|c|}{ Overall: } & 211 & 239 & $47 \%$ \\
\hline
\end{tabular}

We note that overall compliance rates in the 45$50 \%$ range does not seem encouraging for a selfreporting mHealth solution. 40 of 76 patients showed a compliance rate on weekly activities at or above $50 \%$, and similarly 35 of the 76 on daily activities. We do not find this to be all that surprising, as the average compliance rates we are using are dragged down by a significant number of low-weighted compliance rates. That is, patients tend to be compliant or non-compliant from the outset of the study, with enhanced features and technology only moderating the rate of decay in compliance among initial compliant users.

The overall intent of the clinical trial was to construct a model for predicting patient readmission based on data collected from regularly administered PROMIS surveys. While this paper does not report on these clinical results, it is crucial in these types of studies that patients properly engage with the selfreporting mechanism. Therefore, improvements in patient engagement leading to greater protocol 
adherence are of significant importance to the data collection and model formation processes.

\section{Conclusions and Future Work}

This paper presented the next evolution of a pain reporting and management platform for sickle-cell disease, and results on compliance rates tied to time and enhanced app features from a clinical field trial. The work shows some slight improvements in rate of decay from what is accepted in the field and requires more in-depth analysis of the impacts of enhanced features like gamification to make a causal connection.

We are in the process of generalizing the platform to support other chronic conditions that may benefit from self-reporting and care provider monitoring, in areas such as epilepsy in middle school youth, and diabetes and tuberculosis co-morbidities in adults.

\section{References}

[1] Albert, Bandura. "Social foundations of thought and action: A social cognitive theory." NY: Prentice-Hall (1986).

[2] Deterding, Khaled. "Nacke, ua (2011) Gamification: Toward a Definition ${ }^{3}$." CHI 2011 Gamification Workshop Proceedings. Vancouver, BC, Canada.

[3] Deterding, Sebastian, et al. "Gamification. using gamedesign elements in non-gaming contexts." CHI'11 Extended Abstracts on Human Factors in Computing Systems. ACM, 2011.

[4] Johnson, John A. "Ascertaining the validity of individual protocols from web-based personality inventories." Journal of research in personality 39.1 (2005): 103-129

[5] Shah, Nirmish, Jude Jonassaint, and Laura De Castro. "Patients welcome the sickle cell disease mobile application to record symptoms via technology (SMART)." Hemoglobin 38.2 (2014): 99-103.

[6] Smith, Wally R., et al. "Daily assessment of pain in adults with sickle cell disease." Annals of internal medicine 148.2 (2008): 94-101.

[7] Union IT (2010) The world in 2010: ICT facts and figures. International Telecommunication Union.

[8] Archer, Robert P., and David E. Elkins. "Identification of random responding on the MMPI-A." Journal of Personality Assessment 73.3 (1999): 407-421.

[9] Dan, Oana M., and Jennie W. Lai. "How am I doing? The effects of gamification and social sharing on user engagement." Proc. 68th Ann. Conf. American Assoc. for Public Opinion Research. 2013.

[10] Meremikwu, M. M., \& Okomo, U. (2011). Sickle cell disease. Clinical evidence (London : 2006).
[11] Ballas, S. K. (2007). Current Issues in Sickle Cell Pain and Its Management. Hematology 2007, 97-105.

[12] Frei-Jones, M. J., Field, J. J., \& DeBaun, M. R. (2009 April). Risk Factors for Hospital Readmission within 30-Days: A New Quality Measure for Children with Sickle Cell Disease. Pediatr Blood Cancer.

[13] Leschke, J., Panepinto, J. A., Nimmer, M., Hoffman, R. G., Yan, K., \& Brousseau, D. C. (2012). Outpatient Follow-Up and Rehospitalizations for Sickle Cell Disease Patients. Pediatr Blood Cancer, 406-409.

[14] Ballas, S. K. (2011). UPDATE ON PAIN MANAGEMENT IN SICKLE CELL DISEASE. INTERNATIONAL CONFERENCE ON HEMOGLOBIN DISORDERS (pp. 520-529). Kuwait: Jefferson Dgital Commons.

[15] Panepinto, J. A., \& Bonner, M. (2012). Health-Related Quality of Life in Sickle Cell Disease: Past, Present, and Future. Pediatr Blood Cancer, 377-385.

[16] Booker, M. J., Blethyn, K. L., Wright, C. J., \& Greenfield, S. M. (2006). Pain management in sickle cell disease. Chronic Illness, 39-50.

[17] Crosby, L., Modi, A. C., Lemanek, K. L., Guilfoyle, S. M., Kalinyak, K. A., \& Mitchell, M. J. Perceived Barriers to Clinic Appointments for Adolescents with Sickle Cell Disease. J Pediatr Hematol Oncol, 571-576.

[18] Lenhart, A. (2015). Teens, Social Media \& Technology Overview 2015. Pew Research Center. 2009.

[19] Ryan, Richard M., and Edward L. Deci. "Intrinsic and extrinsic motivations: Classic definitions and new directions." Contemporary educational psychology 25.1 2000.

[20] Habgood, MP Jacob, and Shaaron E. Ainsworth. "Motivating children to learn effectively: Exploring the value of intrinsic integration in educational games." The Journal of the Learning Sciences 20.2: 169-206, 2011.

[21] Han, Jennifer, and Chris Barker. "Comparison of Intrinsically Motivating Factors in Educational Games." http://jenniferchristinehan.com/assets/img/egi/Literature Review.pdf, last accessed September 4, 2018.

[22] Preist, Chris, and Robert Jones. "The use of games as extrinisic motivation in education." Proceedings of the 33rd Annual ACM Conference on Human Factors in Computing Systems. ACM, 2015.

[23] Heath, C., Baron, T., Gary, K. and Amresh, A. "Reflection on Assumptions from Designing FemaleCentric Educational Games", Proceedings of the 2nd International Conference on Serious Games, Brisbane, Australia, September 2016.

[24] Gibson, David, Nathaniel Ostashewski, Kim Flintoff, Sheryl Grant, and Erin Knight.,"Digital badges in education." Education and Information Technologies 20.2 : 403-410, 2015.

[25] Gary, K., Stoll, R., Rallabhandi, P., Patwardhan, M., Hamel, D., Amresh, A., Pina, A., Cleary, K. and Quezado, Z. (2017). "MHealth Games as Rewards: Incentive or Distraction?" Proceedings of the ACM International Conference on Digital Health, London UK, July 2017. 